Caries Res. 1978;12:339-352

\title{
ORCA Membershiplist
}

\section{Australia}

Bulgaria

Martin, Prof. N. D. University of Sydney Faculty of Dentistry 2 Chalmers Street Sydney

Datschev, Dr. B.

Medical Academy Sofia

Dental School

1 Georgi Sofiiski str.

Sofia

\section{Austria}

Binder, Dr. K. Geyergasse 12 A-1180Wien

Payer, Dr. W. Am Ring 9/IП A-8010 Graz 9

Wagenbichler, Frau Dr. H. Mozartplatz 244 A-5640 Badgastein

Belgium

Joachim, Dr. M. Rue de Treves 67 B-1042 Brussels 4

Canada

Biswas, Dr. S. University of Manitoba Faculty of Dentistry Dept. of Oral Biology Winnipeg, R3E OW3

Bowden, Prof. G. Dept. of Oral Biology Faculty of Dentistry University of Manitoba 780

Bannatyne Avenue Winnipeg, R3E OW3

Hargreaves, Prof. J. A. Faculty of Dentistry 124 Edward Street Toronto 101

Pourtois, Prof. M.

Université Libre de Bruxelles Czechoslovakia

Faculté de Medecine et de Pharmacie

Service d'Anatomie et d'Embryologie Humaine Kostlan, Dr. J.

Rue de Laines $97 \quad$ Kamenicka

B-1000 Brussels Prague 7

340

ORCA Membershiplist

Ruzicka, J. A.

The Institute of Dental Research

Vinohradska 48

12060 Prague 2

Denmark

Fejerskov, Prof. O.

Dept. of Dental Pathology and Operative Dentistry

Royal Dental College 
Vennelyst Boulevard

DK-8000 Aarhus C

Kilian, Dr. M. Dept. of Microbiology Royal Dental College Vennelyst Boulevard DK-8000

Aarhus C

Kirkegaard, Dr. E.

Dept. of Pedodontics and Preventive Dentistry

Royal Dental College

Vennelyst Boulevard

DK-8000 Aarhus C

Larsen, Dr. M. J.

Dept. of Dental Pathology and Operative Dentistry

Royal Dental College

Vennelyst Boulevard

DK-8000 Aarhus C

Lind, Dr. O. P.

Institute of Social \& Behavioural Sciences

School of Dentistry

Vennelyst Boulevard

DK-8000 Aarhus C

Lindemann, Dr. J. Royal Dental College Jagtvej 160 DK-2100 Copenhagen

Mikkelsen, Dr. L. Dept. of Oral Diagnosis Royal Dental College Vennelyst Boulevard DK-8000

Aarhus C

Møller, Prof. I. J. Olgasvej 9 DK-2970 Hörsholm

Poulsen, Dr. S.

Dept. of Pedocontics and Preventive Dentistry

Royal Dental College

Vennelyst Boulevard

DK-8000 Aarhus C

Theilade, Dr. E. Dept. of Microbiology Royal Dental College Vennelyst Boulevard DK-8000

Aarhus C

Thylstrup, Dr. A.

Dept. of Dental Pathology and Operative Dentistry

Royal Dental College

Vennelyst Boulevard

DK-8000 Aarhus C

Christensen, Dr. J. (Junior Member)

Dept. Dental Pathology and Operative Dentistry

Royal Dental College

Vennelyst Boulevard

DK-8000 Aarhus C

Federal Republic of Germany

Ahrens, Prof. G.

Klinik und Poliklinik für

Zahn-, Mund- und Kieferkrankheiten

Georg Voigt Strasse 3

D-3550 Marburg a. d. Lahn 
Büttner, Prof. W.

Poliklinik für Zahn-, Mund- und Kieferkrankheiten

der Wilhelms Universität

Robert-Koch-Strasse 27a

D-4402 Münster

Gabriel, Dr. E. Zahnklinik Universität Würzburg Pleicherwall 2 D-8700 Würzburg

Ermin, Prof. H. Elektronen Mikroskopie Technische Universität Berlin Strasse des 17. Juni 112, Ernst-Reuter Haus D-1000 Berlin 12

ORCA Membershiplist

341

Gehring, Prof. F. Pleicherwall 2 D-8700 Würzburg

Gülzow, Prof. H. J. Universitätszahnklinik Martinistrasse 52 D-2000 Hamburg 20

Hafer, Frau, H., Pharm.

Bebelstrasse 45

D-6500 Mainz-Bretzenheim

Harndt, Prof. R.

Freie Universität Berlin

Poliklinik und Klinik für

Zahn-, Mund- und Kieferkrankheiten

Assmannshauserstrasse 4-6

D-1000 Berlin 31

Karle, Dr. E.

Klinik für Zahn-, Mund- und Kieferkrankheiten

Pleicherwall 2

D-8700 Würzburg

Knappwost, Prof. A.

Institut für Physikalische Chemie

Laufgraben 24

D-2000 Hamburg 13

Kröncke, Prof. A.

Klinik und Poliklinik für Zahn-, Mund- und

Kieferkrankheiten

Glückstrasse 11

D-8520 Erlangen

Lammers, Prof. T. Hygiene Institut D-46 Dortmund

Lenz, Prof. H. Institut für Kariesforschung Assmannshauserstrasse 4-6 D-1000 Berlin 31

Mierau, Dr. H. D.

Klinik für Zahn-, Mund- und Kieferkrankheiten

Pleicherwall 2

D-8700 Würzburg

Motsch, Prof. A. Geitstrasse 11 D-3400 Göttingen

Naujoks, Prof. R.

Klinik für Zahn-, Mund- und Kieferkrankheiten

Pleicherwall 2

D-8700 Würzburg 
Newesely, Prof. H.

Freie Universität Berlin

Poliklinik und Klinik für Zahn-, Mund- und

Kieferkrankheiten

Assmannshauserstrasse 4-6

D-1000 Berlin 31

Niedenthal, Dr. A. M edizinaldirektor Dreieichring 24 Stadtgesundheitsamt D-6050

Offenbach/Main

Patz, Dr. J.

Poliklinik und Klinik für Zahn-, Mund- und

Kieferkrankheiten

Pleicherwall 2

D-8700 Würzburg

Ranke, Prof. B.

Poliklinik und Klinik für Zahn-, Mund- und

Kieferkrankheiten

Krankenhaus Eppendorf

Martinistrasse 52

D-2000 Hamburg 20

Ranke, Frau Dr. E.

Klinik und Poliklinik für Zahn-, Mund- und

Kieferkrankheiten

Universitätskrankenhaus Eppendorf

Martinistrasse 52

D-2000 Hamburg 20

Riethe, Prof. P.

Klinik und Poliklinik für Zahn-, Mund- und

Kieferkrankheiten

Osianderstrasse 2-8

D-7400 Tubingen 1

Rotgans, Dr. J.

Universität Tubingen

Klinik und Poliklinik für Zahn-, Mund- und

Kieferkrankheiten

Osianderstrasse 2-8

D-7400 Tubingen 1

342

ORCA Membershiplist

Schmidt, Prof. H. J. Leonorenstrasse 25 D-7000 Stuttgart-Degerloch

Schübel, Prof. F. Westdeutsche Kieferklinik Moorenstrasse 5 D-4000 Düsseldorf 1

Trautner, Dr. K. Pleicherwall 2 D-8700 Würzburg

Vahl, Prof. J.

Institut für Medizinische Physik

Westfälische Wilhelmsuniversität

Ruefferstrasse 68 
D-44 Münster

Finland

Larmas, Dr. M. Institute of Dentistry University of Turku SF-20520 Turku 52

Luoma, Prof. H. Dept. of Dentistry University of Kuopio P.O. Box 138 SF-70101 Kuopio 10

Mäkinen, Prof. K. K. Institute of Dentistry University of Turku SF-20520 Turku 52

Paunio, Prof. I.

University Institute of Dentistry

Fabíaninkatu 24

SF-00100 Helsinki 10

Scheinin, Prof. A. Institute of Dentistry University of Turku SF-20520 Turku 52

Tenovuo Dr. J. (Junior Member) Institute of Dentistry University of Turku SF-20520 Turku 52

France

Frank, Prof. R.

Institut de Chirurgie Dentaire

Faculté de Médecine

1, Place de ГHôpital

F-67000 Strasbourg

Goldberg, Dr. M.

Lab. d'Embryologie et Histologie Dentaire

Université Rene Descartes

Faculté de Chirurgie Dentaire

1, Rue Maurice Arnoux

F-92120 Montrouge

Triller, Dr. R. Z. M. Université Rene Descartes Faculté de Chirurgie Dentaire 1, Rue Maurice Arnoux F-92120 Montrouge

Weill, Prof. R.

96, Boulevard Malesherbes

F-75017 Paris

Franquin, Dr. J. C. Docteur en Médecine Dentaire de ГUniversité de Geneve 230 Avenue du

Prado F-13008 Marseille

German Democratic Republic

Auermann, Dr. E.

Forschungsstelle des Bezirks-Hygiene-Instituts

Gustav-Adolph-Strasse 18

DDR-90 Karl-Marx-Stadt

Künzel, Prof. W. Präventive Stomatologie Sektion Stomatologie Medizinische Akademie Erfurt Nordhäuser Strasse 74 DDR-50 Erfurt

Maiwald, Dr. H.-J. Präventive Stomatologie Sektion Stomatologie Medizinische Akademie

Erfurt Nordhäuser Strasse 74 DDR-50 Erfurt

ORCA Membershiplist

343

Pilz, Prof. W.

Klinik und Poliklinik für Stomatologie

Abteilung für Konservierende Stomatologie

Medizinische Akademie Dresden 
Fiedlerstrasse 25

DDR-8019 Dresden

Schmidt, Doz. Dr. Dr. H.

Physiologisch-Chemisches Institut Martin-Luther-Universität Halle Hollystrasse 1 DDR-402

Halle

Schützmannsky, Frau Prof. G. Jugendzahnklinik Harz 42-44 DDR-402 Halle

Stösser, Dr. L. (Junior Member) Präventive Stomatologie Sektion Stomatologie Medizinische

Akademie Erfurt Nordhäuser Strasse 74 DDR-50 Erfurt

Taatz, Prof. H.

Klinik und Poliklinik für Stomatologie

Abteilung für Konservierende Stomatologie

Martin-Luther-Universität Halle

Grosse Steinstrasse 19

DDR-402 Halle

Wegner, Prof. $\mathrm{H}$.

Poliklinik für Konservierende Stomatologie

Karl-Marx-Universität Leipzig

Nürnbergerstrasse 57

DDR-701 Leipzig

Great Britain

Armstrong, Prof. W. G. Royal Dental School Leicester Square London WC2H 7LJ

Bowen, Dr. W. H.

United States Public Health Service

National Institute for Dental Research

Westwood Buildings

Bethesda, MD 20014 (USA)

Bradford, Prof. E. Dental School of the University Lower Maudlin Street Bristol 1

Cornic, Dr. D. E. R. Institute of Dental Surgery Eastman Dental Hospital Gray's Inn Road

London WC1

Crabb, Prof. H. S. M. Dental School and Hospital Clarendon Way Leeds LS2 9LU

Darling, Prof. A. I.

Dental School of the University

Lower Maudlin Street

Bristol

Davies, Dr. T. G. H. Messrs. Colgate-Palmolive Ltd. Ordsall Lane Salford M5 3FS

Duff, Dr. E. J. Turner Dental School Bridgeford Street Manchester M15 6FH

Eastoe, Dr. J. Dept. of Dental Science Royal College of Surgeons Lincoln's Inn Fields London

WC2A 3PN

Edgar, Dr. W. M.

University of Newcastle upon Tyne

The Dental School

Dept. of Oral Physiology

Framlington Place

Newcastle upon Tyne NE2 4BW

Ferguson, Dr. D. B. Dept. of Physiology Medical Faculty Stopford Building Oxford Road

Manchester 13 
ORCA Membershiplist

Geddes, Dr. D. A. M.

Dept. of Oral Pathology

Glasgow Dental Hospital and School

378 Sauchiehall Street

Glasgow G2 3JZ

Green, Dr. R. M.

Welsh National School of Medicine

University of Wales

Dental School

Heath, Cardiff CF4 4XY

Grenby, Dr. A. H.

Dept. of Oral Medicine and Pathology Guy's Hospital Medical College London SE1 9RT

Hallett, Prof. G. E. Newcastle upon Tyne University Sutherland Dental School Northumberland

Road Newcastle upon Tyne 1

Hallsworth, Dr. A. S. University of Leeds School of Dentistry Dept. of Oral Biology Clarendon Way Leeds LS2 9LU

Hardwick, Prof. J. L. Turner Dental School Bridgeford Street Manchester M15 6FH

Hartles, Prof. R. L. School of Dentistry P.O. Box 147 Liverpool L 69 3BX

Ingram, Dr. G. S. Unilever Research Laboratory 455 London Road Isleworth, TW7 5AB

Jackson, Prof. D. School of Dentistry Clarendon Way Leeds LS2 9LU

James, Prof. P. M. C. The Dental School St. Mary's Row Birmingham B4 6NN

Jenkins, Prof. G. N. Dept. of Oral Physiology Framlington Place Newcastle upon Tyne NE2

4BW

Johnson, Prof. N. W.

Dept. of Oral Pathology

The London Hospital Medical College

Turner Street

London E1 2AD

Joyston-Bechal, Dr. S.

Dept. of Oral Medicine

London Hospital Medical College

Turner Street

London E1 2AD

Leach, Dr. S. A.

School of Dentistry, Dept. of Dental Sciences

P.O. Box 147

Liverpool L69 3BX

Levine, Dr. R. S. Dept. of Oral Medicine Turner Dental School Bridgeford Street Manchester M15 6FH

Machlachlan, Dr. J. S. Buxton Lodge Buxton Road 32 Weymouth DT4 9PJ

Miller, Prof. J.

The Dental School

Heath 


\section{Cardiff CF4 4XY}

Mortimer, Prof. K. V.

Royal Dental Hospital and School

Dental Surgery

Leicester Square

London WC2H 7LJ

ORCA Membershiplist

345

Nixon, Prof. G. S. Dept. of Conservative Dentistry Turner Dental School Bridgeford Street Manchester M15 6FH

O’Mullane, Dr. D. M. Chief Dental Officer Department of Health Hawkins House Dublin 2

Prout, Dr. R. E. S. Dept. of Biochemistry The University Sheffield S10 2TN

Puttnam, Dr. N. Colgate-Palmolive Ltd. 373 Ordsall Lane Salford 5 Lanes.

Robinson, Dr. C. School of Dentistry Dept. of Oral Biology Clarendon Way Leeds LS2 9LU

Rugg-Gunn, Dr. A.

Dept. of Oral Physiology

University of Newcastle upon Tyne

The Dental School

Framlington Place

Newcastle upon Tyne NE2 4BW

Speirs, Prof. R. L.

Dept. of Physiology

The London Hospital Medical College

Turner Street

London E1 2AD

Stephen, Dr. K. W.

Glasgow Dental Hospital and School

Dept. of Oral Medicine and Pathology

378 Sauchiehall Street

Glasgow G2 3JZ

Sutcliffe, Dr. P. Edinburgh Dental Hospital 31 Chambers Street Edinburgh EH1 1JA

Tatevossian, Dr. A. University College P.O. Box 78 Cardiff CF1 1XL

Wagg, Dr. B. J.

Unilever Research Laboratories 455 London Road Isleworth, TW7 5AB

Wallis, Dr. C. P. Dept. of Dental Surgery University of Edinburgh 31 Chambers Street

Edinburgh EH1 1JA

Weatherell, Dr. J. A. University of Leeds School of Dentistry Dept. of Oral Biology Clarendon

Way Leeds LS2 9LU

Winter, Prof. G. B. Eastman Dental Hospital Gray’s Inn Road London WC1

Beeley, Dr. J. A.

Glasgow Dental Hospital and School

University of Glasgow

Dept. of Dental Biochemistry

378 Sauchiehall Street

Glasgow G2 3JZ 
Boyde, Dr. A. Department of Anatomy University College London Gower Street London WC1 Donoghue, Dr. H. (Junior Member) School of Studies in Medical Sciences University of Bradford Bradford BD7 1DP

Hardie, Dr. J. M.

The London Hospital Medical College

Dental School

Turner Street

London E1 2AD

346

ORCA Membershiplist

Howat, Dr. A. P. (Junior Member)

University of Brimingham

Dept. of Children's Dentistry \& Orthodontics

Dental School

St. Chad's Queensway

Birmingham B4 6NN

Kidd, Dr. E. A. M.

Royal Dental Hospital of London

School of Dental Surgery

Leicester Square

London WC2H 7LJ

Mageean, Dr. J. F. University College of Cork Lee Mills House Prospect Row Cork (Ireland)

Murray, Prof. J. J.

Dept. of Child Dental Health

Dental School

University of Newcastle upon Tyne

Northumberland Road

Newcastle upon Tyne NE1 8TA

Poole, Dr. G. F. D.

Medical Research Council Dental Research Unit The Dental School Lower Maudlin Street

Bristol BS1 2LY

Hungary

Adler, Prof. P.

Stomatologische Universitätsklinik

H-4012 Debrecen

Bánóczy, Prof. J.

Clinic of Conservative Dentistry

Semmelweis Medical University

Mikszáth K. tér 5

H-1088 Budapest

Bruszt, Dr. P. Attila utca 6 H-6500 Baja

Csobán, Dr. G. DOTE Ed Szerv. Int. H-4012 Debrecen

Hattyasy, Prof. D. Maros u. 23. III.4. H-1122 Budapest

Tóth, Dr. A.

Stomatologische Universitätsklinik 
H-4012 Debrecen

Tóth, Prof. K.

University Medical School

Dept. of Dentistry and Oral Surgery

Lenin kòrút 64

H-6720 Szeged

Zelles, Dr. T. Semmelweis University Dept. of Physiopatology Medical School Hógyes Endre u.

9 H-1092 Budapest

Israel

Gedalia, Prof. I.

The Hebrew University

Hadassah Medical School

Preventive Dentistry

P.O. Box 1172

Jerusalem

Deutsch, Dr. D.

Dept. of Preventive Dentistry

The Hebrew University

Hadassah Medical School

P.O. Box 1172

Jerusalem

Italy

Baratieri, Prof. A.

Direttore Istituto di Clinica Odontoiatrica

Facoltà di Medicina e Chirurgia

Universita Cattolica del Sacro Cuore

Largo A. Gemelli, 8

1-00168 Roma

ORCA Membershiplist

347

Capozzi, Prof. L. Clinica Odontoiatrica Università degli Studi Viale Regina Elena 287/A 100161 Roma

Grippaudo, Prof. G. University of Rome Dental Clinic Viale Regina Elena 287/A 1-00161 Roma Marci, Prof. F. Via Roma 1 1-02100 Rieti

Rose, Dr. M.

Via di Porta Pinciana 4

1-00187 Roma

Sforzolini-Scassellati, Prof. Cas. Post. 324 1-06100 Perugia

Singer, Prof. F.

Istituto Odontostomatologico

Via Piave 8

1-39012 Merano

Japan

Minoguchi, Prof. Kyoto University Dept. of Stomatology Kyoto

Luxembourg 
Klees, Dr. L.

17, Boulevard Royal

Luxembourg

The Netherlands

Arends, Prof. J. Lab. voor Materia Technica Rijksuniversiteit Groningen Antillenstraat 11-13

NL-9714 JT Groningen

Backer-Dirks, Prof. O. (Honorary Member) Afd. Preventieve Tandheelkunde Rijksuniversiteit Utrecht Sorbonnelaàn 16 NL-3584 CA Utrecht

Bartheld, Dr. F. von Afd. Caries Pathogenese Tandheelkunde Rijksuniversiteit Utrecht

Sorbonnelaàn 16 NL-3584 CA Utrecht

Driessens, Prof. F. C. M.

Afd. Tandheelkundige Materialen

Katholieke Universiteit

Medische Faculteit

Postbus 9101

NL-6500 HB Nijmegen

's-Gravenmade, Dr. E. Lab. voor Materia Technica Rijksuniversiteit Groningen Antillenstraat 11-13 NL-9714 JT Groningen

Groeneveld, Dr. A. Lab. voor Microbiologie Catharijnesingel 59 NL-3511 GG Utrecht

Houwink, Prof. B.

Afd. Preventieve en Sociale Tandheelkunde

Vrije Universiteit

de Boelelaan 1115

NL-1081 HV Amsterdam

Huis in 't Veld, Dr. J. H. J. Afd. Preventieve Tandheelkunde Rijksuniversiteit Utrecht

Sorbonnelaàn 16 NL-3584 CA Utrecht

König, Prof. K. G.

Institute of Preventive and Community Dentistry

The University Medical Faculty

Postbus 9101

NL-6500 HB Nijmegen

Kwant, Dr. G. W. Edeseweg 51 NL-6721 JP Bennekom

348

ORCA Membershiplist

Myrberg, Prof. N. E. A. Universiteit van Amsterdam Afd. Orthodontie Louwesweg 1 NL-1066 EA Amsterdam

Palenstein Helderman, Dr. W. H. van Afd. Preventieve Tandheelkunde Ríjksuniversiteit Utrecht Sorbonnelaàn 16 NL-3584 CA Utrecht

Perdok, Prof. W. G. Instituut voor Kristalfysica Melkweg 1 NL-9718 EP Groningen

Plasschaert, Prof. A. J. M.

Afd. Conserverende Tandheelkunde

voor Volwassenen

Katholieke Universiteit

Medische Faculteit

Postbus 9101 
NL-6500 HB Nijmegen

Stoppelaar, Dr. J. D. de

Afd. Preventieve Tandheelkunde

Rijksuniversiteit Utrecht

Sorbonnelaàn 16

NL-3584 CA Utrecht

Wöltgens, Dr. J. H. M.

Afd. Preventieve en Sociale Tandheelkunde

Vrije Universiteit

de Boelelaan 1115

NL-1081 HV Amsterdam

New Zealand

Featherstone, J. D. B.

Victoria University of Wellington

Chemistry Department

Private Bag

Wellington

Norway

Birkeland, Dr. J. M. University of Oslo Dental Faculty Geitmyrsveien 71 Blindern N-Oslo 4

Bjorvatn, Dr. K. University of Bergen School of Dentistry Aarstadveien 17 N-5000 Bergen

Eeg-Larsen, Prof. N. University of Oslo Dental Faculty Geitmyrsveien 71 Blindern N-Oslo 4

Eriksen, Dr. H. M. University of Oslo Dental Faculty Geitmyrsveien 71, Blindern

N-Oslo 4

Fehr, Prof. F. R. von der University of Oslo Dental Faculty Geitmyrsveien 71, Blindern N-Oslo 4 Hagen, Prof. A. R. Reistadlia 23 N-1364 Hvalstad

Hals, Prof. E. University of Bergen School of Dentistry Aarstadveien 17 N-5000 Bergen

Mörch, Prof. T. University of Bergen School of Dentistry Aarstadveien 17 N-5000 Bergen

Rólla, Prof. G. University of Oslo Dental Faculty Geitmyrsveien 71, Blindern N-Oslo 4

Sönju, Dr. T. University of Oslo Dental Faculty Geitmyrsveien 71, Blindern N-Oslo 4

ORCA Membershiplist

349

Spain

Izel, Dr. N. C. Odontologue Navas de Tolosa 27 Pamplona

Sweden

Angmar-Mansson, Dr. B. School of Dentistry Karolinska Institutet Box 3207 S-103 64

Stockholm 3

Ekstrand, Dr. J. (Junior Member)

Karolinska Institutet

Odontologiska Klinikerna, Dept. of Cariology

Box 4064

S-141 04 Huddinge

Ericson, Prof. T. Odontologiska Fakulteten S-901 87 Umea 6

Ericsson, Prof. Y. Karolinska Institutet P.O. Box 3207 S-103 64 Stockholm 3

Forsman, Dr. O. B. Avd. for Tandhaelsovard Centrallasarettet S-351 85 Vaexjö 
Frostell, Prof. G. Dept. of Cariology Faculty of Odontology Karolinska Institutet P.O. Box 3207 S-103 64 Stockholm 3

Grahnén, Prof. H. University of Umea School of Dentistry Dept. of Pedodontics Norrlandsgatan 18 b S-90590 Umea 1

Granath, Prof. L. E. University of Lund School of Dentistry S-214 21 Malmö

Koch, Dr. G.

Institute for Post Graduate Dental Education

Barnarpsgatan 39

S-552 56 Jönköping 1

Krasse, Prof. B.

University of Göteborg

Faculty of Odontology

Fack

S-400 33 Göteborg

Larje, Dr. O. Svinninhegrånd 11 S-163 61 Spånga

Söremark, Prof. R. Karolinska Institutet School of Dentistry P.O. Box 3207 S-103 64 Stockholm 3

Torell, Dr. P. Friggagatan 25 A S-416 64 Göteborg C

Petersson, Dr. L. G. Odontologiska Institutionen Barnarpsgatan 39 S-552 56 Joenkoeping 1

Johnson, Dr. G. Karolinska Institutet Dept. of Cariology P.O. Box 3207 S-103 64 Stockholm 3

Månsson, Dr. B.

Head of Public Dental Health

County of Våsterbotten

Norrlandgatan 18 B

S-902 48 Umea

Svanberg, Dr. M. Dept. of Cariology Odont. Clinic University of Göteborg S-400 33 Göteborg 33

Switzerland

Baud, Prof. C. A. Institut de Morphologie Ecole de Médecine Rue de ГEcole de Médecine CH-

1205 Geneva

350

ORCA Membershiplist

Baume, Prof. L. J. Institut de Médecine Dentaire 19, Rue Barthélemy-Menn CH-1211 Geneva 4 Bäumler, Dr. chem. J. Gerichtschemiker Polizeidepartement Basel 22 Pestalozzistrasse CH-4056 Basel

Cimasoni, Prof. G. Institut de Médecine Dentaire 19, Rue Barthélemy-Menn CH-1211 Geneva 4 Dustin, Prof. J. P.

Moulinsart

CH-1295 Tannay-Mies

Fiore-Donno, Prof. S. Institut de Médecine Dentaire 19, Rue Barthélemy-Menn CH-1211

Geneva 4

Graf, Prof. H.

Zahnärztliches Universitätsinstitut

Abt. für Parodontologie

Freiburgstrasse 7 


\section{CH-3010 Bern}

Guggenheim, Prof. B. Universität, Zahnärztliches Institut Postfach Zurich 28 CH-8028 Zurich Held, Dr. H. R. (Honorary Member) Chalet «le Refuge» CH-1264 St. Cergue

Hotz, Prof. P. Klinik für Zahnerhaltung Universität Bern Freiburgstrasse 7 CH-3012 Bern

Hürny, Dr. T. Spitalgasse 4 CH-3010 Bern

Marthaler, Prof. Th. Zahnärztliches Universitätsinstitut Plattenstrasse 11 Postfach Zurich $28 \mathrm{CH}-$ 8028 Zurich

Mühlemann, Prof. H. R. Zahnärztliches Universitätsinstitut Plattenstrasse 11 Postfach Zurich 28

CH-8028 Zurich

Schroeder, Prof. A. Zahnärztliches Universitätsinstitut Freiburgstrasse 7 CH-3010 Bern

Schroeder, Prof. H. E. Zahnärztliches Universitätsinstitut Plattenstrasse 11 Postfach Zurich 28

CH-8028 Zurich

Somogyi, Prof. J. C.

Institut für Ernährungsforschung

Seestrasse 72

CH-8803 Rüschlikon-Zürich

Sutter, Dr. P. E. Falknerstrasse 12 CH-4051 Basel

Wespi, Dr. H. J. Sonneggweg CH-5034 Suhr

Bang, Dr. S. 31, Rue Dancet CH-1205 Geneva

Hefti, Dr. A. (Junior Member) Zahnärztliches Universitätsinstitut Petersplatz 14 CH-4051 Basel

USA

Bibby, Prof. B. G. (Honorary Member) Eastman Dental Dispensary University of Rochester Main Street East 800 Rochester, NY 14603

ORCA Membershiplist

351

Brudevold, Prof. F. Forsyth Dental Center 140 the Fenway Boston, MA 02115

Carlos, Dr. J. P. National Institutes of Health National Institute for Dental Research Bethesda, MD 20014

Curzon, Dr. M. E. J. Dept. of Caries Research Eastman Dental Center 625 Elmwood Avenue Rochester, NY 14620

Glass, Dr. R. L. Forsyth Dental Center 140 The Fenway Boston, MA 02115

Gray, Dr. J. A. The Proctor \& Gamble Co. Miami Valley Laboratories P.O. Box 39175

Cincinnati, OH 45239

Hefferen, Dr. J.

Amer. Dental Association, Health Foundation

211 E. Chicago Avenue

Chicago, IL 60611

Horowitz, Dr. H. S.

Chief Community Programs Section

Caries Prevent. \& Research Branch

NIDR-NIH

Bethesda, MD 20014

Knutson, Dr. J. W. School of Dentistry University of California Medical Center Los Angeles, CA 90024 
Meckel, Dr. A. H. The Proctor \& Gamble Co. Miami Valley Laboratories P.O. Box 39175

Cincinnati, OH 45239

Miller, Prof. Wm. A.

State University of New York at Buffalo

Health Science Center

Dept. of Oral Biology

4510 Main Street

Buffalo, NY 14226

Muhler, Prof. J. C.

Preventive Dentistry Research Institute 2101 Coliseum Boulevard East Fort Wayne IN 46805

Newbrun, Prof. E.

School of Dentistry

Dept. of Oral Medicine and Hospital Dentistry

University of California

San Francisco, CA 94143

Nylen, Dr. M. U.

N.I.D.R.

National Institute of Health

Bethesda, MD 2001

Silverstone, Prof. L. M. Division of Cariology College of Dentistry University of Iowa Iowa City, IA 52242

Wei, Prof. S. H. Y. University of Iowa College of Dentistry Dept. of Pedodontics Iowa City, IA 52242

Yugoslavia

Stosic, Prof. P. Universität Zarka Pucara 23 Belgrade

Tomic, Dr. D.

Pharmakolog. Institut der Medizinischen Fakultät

Salata 11

Zagreb

Vrbic, Dr. V. Stomatologische Klinik Zaloska C2 61105 Ljubliana

Topic, Prof. B. Clinic of Oral Diseases Faculty of Stomatology Dental School University of

Sarajevo Sarajevo

352

ORCA Membershiplist

Corporate Members

Akzo Consumenten Produkten Nederland B.V.

25 Fruitweg

P.O. Box 1196

N-2500 BD 's-Gravenhage (The Netherlands)

Rep.: Dr. H. Hoogendoorn, Director

Benzon, Alfred A/S Direction

29 Halmtorvet

DK-1700 Copenhagen (Denmark) Rep.: Mrs. E. Kundsen

Blendax-Werke Postfach 1580 D-6500 Mainz (FRG) Rep.: Dr. L'Orange

Gebr. Broschek G. K. G. A-6391 Fieberbrunn (Austria) 
Colgate Palmolive Ltd.

76 Oxford Street

London W1A 1EN (Great Britain)

Rep.: Dr. T. G. H. Davies

Elida Cosmetic SA Förrlibuckstrasse 10 Postfach, 8031 Zurich CH-8031 Zurich (Switzerland)

Rep.: Mr. H. J. Ramser

Elida-Gibbs GmbH Postfach 500449 D-2000 Hamburg 50 (FRG) Rep.: Dr. M. Ermerth

Gaba A. G.

Grabetsmattweg

CH-4106 Therwil (Switzerland)

Rep.: Mr. H. Schmid, Director

Goupil Laboratoires

30 Avenue du President WilsonF-94230 Cachan (France)Rep.: Dr. G. Finidori

Informationskreis für Mundhygiene und

Ernährungsverhalten

Siesmayerstrasse 15

D-6000 Frankfurt am Main (FRG)

Rep.: Mrs. B. von Tobien

Lycasin Starch Ltd.

Box 1077

S-260 90 Bastad (Sweden)

Rep.: Dr. E. Rockström, Director

Pacemaker Corporation 2255 N.E. 194th Portland, OR 97230 (USA) Rep.: S. Deitzman,

President

Proctor \& Gamble Co. Winten Hill Technical Center 6110 Center Hill Road Cincinnati, OH

45224 (USA) Rep.: Dr. R. E. Tenny

Suikerstichting Nederland

Lijnbaansgracht 210

Postbus 7498

1007 JL Amsterdam (The Netherlands)

Rep.: Dr. W. Visser, Director

Unilever Research Ltd.

Isleworth Laboratory

455 London Road

Isleworth, Middlesex TW7 5AB (Great Britain)

Rep.: Dr. G. S. Ingram

Verein für Zahnhygiene E.V. Marktplatz 5 D-6100 Darmstadt (FRG) Rep.: Mr. F. Römer

Xyrofin A. G. Lättichstrasse 8 a CH-6340 Baar (Switzerland) Rep.: Prof. K. F. Gey

Zyma Blaes A.G. Zielstrasse 38 D-8000 München (FRG) Rep.: Dr. Hartel, Director

Zyma-Galen S.A. Rue de Want 209 B-1020 Bruxelles (Belgium) Rep.: Mr. Kuhnen

Zyma Nederland

Industrieweg 42

3641 RM Mijdrecht (The Netherlands)

Rep.: Mr. L. de Lege, Director

Zyma S.A.

C. CH-1260 Nyon (Switzerland) 
Rep.: Mr. I. G. Macpherson, PM 